\title{
Coeficientes da equação de Angström-Prescott e sua influência na evapotranspiração de referência em Seropédica, RJ
}

\author{
Daniel F. de Carvalho ${ }^{1}$, Dione G. da Silva ${ }^{2}$, Adilson P. de Souza ${ }^{3}$, \\ Daniela P. Gomes ${ }^{4} \&$ H ermes S. da Rocha ${ }^{5}$
}

\begin{abstract}
RESU MO
A evapotranspiração de referência (ETo) é dependente da quantidade de energia disponível no ambiente e a radiação solar (Rs) é uma variável importante na sua estimativa. Mediante o exposto, o presente trabalho teve como objetivo determinar os coeficientes " $a$ " e " $b$ " da equação de Angströn-Prescott para Seropédica, RJ, e avaliar seus efeitos na estimativa da Rs e da ETo. U tilizaram-se medidas da radiação solar global e número efetivo de horas de brilho solar da estação Ecologia Agrícola (83741 - -IN M ET) do período de 2000 a 2007. O s coeficientes foram determinados por análise de regressão linear, para todo o período e para períodos mensais e anuais. U tilizando-se os coeficientes calculados e os propostos pela FAO $(0,25$ e 0,50$)$ estimaram-se Rs e ETo, e se avaliaram seus desempenhos. 0 s coeficientes médios mensais "a" variaram de 0,232 a 0,299 e "b" de 0,397 a 0,504. Os coeficientes médios anuais a e $b$ foram 0,295 e 0,417 e os do período geral, 0,282 e 0,433, respectivamente. 0 melhor desempenho observado para a estimativa da Rs e da ETo foi obtido quando se utilizaram os coeficientes médios mensais da equação Angströn-Prescott.
\end{abstract}

Palavras-chave: radiação solar, brilho solar, estação meteorológica, Penman-M onteith

\section{Coefficients of the Angström-Prescott equation and its influence on evapotranspiration in Seropedica, Rio de Janeiro State, Brazil}

\begin{abstract}
The reference evapotranspiration (ETo) is dependent on the amount of energy available in the environment, being solar radiation (SR) as an important variable in its estimation. This study was carried out in order to determine the coefficients " $a$ " and " $b$ " of the Angstron-Prescott equation for Seropédica, Rio de Janeiro State, Brazil, and to evaluate their effects on the SR and ETo estimation. M easures of global solar radiation and effective number of hours of sunshine were used from station Ecology Fund (83741 - IN M ET) for the period of 2000 to 2007. The coefficients were determined by linear regression analysis for the entire period and monthly and yearly periods. U sing the calculated coefficients and those proposed by FAO $(0.25$ and 0.50$)$ SR and ETo were estimated and evaluated their performance. The average monthly coefficients " $a$ " ranged from 0.232 to 0.299 and " $b$ " from 0.397 to 0.504 . Annual mean coefficients " $a$ " and " $b$ " were 0.295 and 0.417 , and for general period were 0.282 and 0.433 , respectively. The best performance observed for the estimated SR and ETo was obtained when the monthly coefficients of the equation Angströn-Prescott were adopted.
\end{abstract}

Key words: solar radiation, sunshine, weather station, Penman-M onteith

${ }^{1}$ DE/U FRRJ. BR 465, km 7. CEP 23890-000, Seropédica, RJ. Fone (21) 2682-1864. E-mail: carvalho@ufrrj.br. Bolsista do CN Pq. ${ }^{2}$ D outoranda em Produção Vegetal, U EN F/CCTA, CEP 28013-602, Campos dos G oytacazes, RJ. Fone (22) 2739-7104. E-mail: dione_galvao@yahoo.com.br ${ }^{3}$ U FMT, Instituto de Ciências Agrárias e Ambientais, Campus de Sinop, Distrito Industrial, CEP 78550-000, Sinop-MT, Brasil. Fone: (66) $3531-1663$. E-mail: adilsonpacheco@ufmt.br

${ }^{4}$ Mestranda em Fitotecnia, U FRRJ, CEP 23890-000, Seropédica, RJ. Fone (21) 3787-3755. E-mail: danielagomesagro@ hotmail.com

${ }^{5} \mathrm{G}$ raduando em Engenharia Agrícola, U FRRJ, CEP 23890-000, Seropédica, RJ. Fone (21) 2682-1864. Bolsista de IC - CN Pq. E-mail: hermesrocha@hotmail.com 


\section{INTRODUÇÃO}

A radiação solar é a fonte de energia primária para os fenômenos meteorológicos, além de ser responsável por todos os processos físicos e bioquímicos que ocorrem no planeta. Tanto a radiação global (Rs) quanto a duração do brilho solar (n) são elementos meteorológicos significativos, que contribuem expressivamente para estudos agronômicos, ecológicos e hidrológicos.

Segundo Almorox et al. (2008), a informação local da radiação solar global, além de ser utilizada nos cálculos de simulação de crescimento das culturas agrícolas pode ser, também, aplicada para delineamento de sistemas alternativos de produção de energia. Dantas et al. (2003) relatam que estudos nos quais se estima o potencial de radiação solar disponível à superfície terrestre, são justificáveis para execução de projetos de irrigação e conservação de alimentos, entre outros.

Com o advento das estações meteorológicas automáticas, foram desenvolvidos sensores e dispositivos de aquisição de dados que têm permitido a obtenção de leituras instantâneas dos elementos meteorológicos e o armazenamento dessas informações. Nessas estações é comum o uso de sensores de radiação solar direta e, desta forma, os dados de insolação normalmente obtidos em estações convencionais, por meio de heliógrafos, deixam de ser obtidos (Blanco \& Sentelhas, 2002). Por outro lado, Liu et al. (2009b) comentam que as estações convencionais normalmente não são equipadas com radiômetros, actinógrafos e piranômetros, impossibilitando a obtenção de uma leitura direta da radiação solar.

Liu et al. (2009b) ressaltam que em 1924 Angstrom propôs uma relação linear entre a razão da radiação solar global média e o seu valor correspondente a um dia completamente claro e a razão entre a duração média diária do brilho solar com a sua máxima duração. Posteriormente, a fim de resolver as dificuldades na obtenção da radiação em dias claros, Prescott sugeriu o uso da radiação solar extraterrestre ou radiação no topo da atmosfera, levando à criação de um novo modelo, conhecido como equação de Angström-Prescott.

A equação de Angström-Prescott pode ser utilizada com base em dados diários, quinzenais, mensais, sazonais e anuais e seus coeficientes ( $a$ e b) devem ser ajustados localmente e para diferentes épocas do ano, a fim de melhor representarem a variação da radiação em função do brilho solar. De acordo com Almorox et al. (2005), os coeficientes dependem da latitude, época do ano e altitude, e variam em função das mudanças no tipo e espessura de nuvens e concentração de poluentes. $\mathrm{O}$ coeficiente "a" representa a radiação difusa da irradiação solar global e o "b", a radiação direta (Dallacort et al., 2004).

Dentre as diversas aplicações da radiação solar global, Almorox et al. (2008) destacam o uso nos modelos de crescimento de plantas e na estimativa da evapotranspiração em projetos de sistemas de irrigação. De acordo com Liu et al. (2009a), informações confiáveis de evapotranspiração (ET) são essenciais em estudos relacionados à hidrologia, ao clima e ao manejo dos recursos hídricos; no entanto, segundo os autores, há poucas informações comparativas entre os coeficientes de Angström-Prescott calibrados localmente e aqueles recomendados pela FAO, e seus efeitos na estimativa da radiação solar global e, consequentemente, na evapotranspiração de referência (ETo). Segundo Allen et al. (1998), a ETo é dependente da quantidade de energia disponível no ambiente, sendo a radiação solar uma importante variável na sua estimativa.

Valores dos coeficientes da equação de Angström-Prescott foram determinados em várias regiões do Brasil: Cervellini et al. (1966) (Estado de São Paulo), Campello Júnior (1998) (Santo Antonio do Leverger-MT), Azevedo et al. (1990) (Juazeiro, BA, Sumé e Souzam, PB), Blanco \& Sentelhas (2002) (Piracicaba, SP); Dantas et al. (2003) (Lavras, MG); Dallacort et al. (2004) (Palotina, PR), Valiati \& Ricieri (2005) (Cascavel, PR), Dornelas et al. (2006) (Brasília, DF), dentre outros. Mediante o exposto se desenvolveu este trabalho a fim de estimar os coeficientes (a e b) da equação de Angström-Prescott e seus efeitos na estimativa da radiação solar global e da evapotranspiração de referência, em Seropédica, RJ.

\section{Material E MÉTODOS}

Realizou-se o estudo a partir de dados meteorológicos das estações convencional (83741) e automática, denominadas Ecologia Agrícola, ambas pertencentes à rede do Instituto Nacional de Meteorologia (INMET), localizadas no município de Seropédica, RJ, nas coordenadas $22^{\circ} 45^{\prime} 13^{\prime}$ 'S e 43 40' 23", $\mathrm{W}$, e apresentando uma altitude de $34,0 \mathrm{~m}$. Segundo a classificação de Köppen, o clima da região é classificado como Aw, com chuvas concentradas no período novembro a março, com precipitação anual média de $1213 \mathrm{~mm}$ e temperatura média anual de $24,5^{\circ} \mathrm{C}$ (Carvalho et al., 2006).

O período de análise dos dados foi de 07/05/2000 (início de operação da estação automática) a 31/12/2007 (término do período de funcionamento da estação convencional). $\mathrm{Na}$ estação convencional se obtiveram os dados diários de insolação (n) e, na automática os dados diários de radiação solar (Rs), além dos dados de temperatura, umidade relativa e velocidade do vento, os quais permitiram, posteriormente, a estimativa da evapotranspiração de referência (ETo). Os dados concernentes à estação automática, foram obtidos em formato digital na Coordenação Geral de Agrometeorologia do INMET. Os dados de insolação (n) foram digitados em planilha eletrônica, possibilitando obter-se, diariamente e para todo o período, a razão $\mathrm{n} / \mathrm{N}$, sendo $\mathrm{N}$ calculado diariamente em função da latitude do local (Allen et al., 1998).

Por meio de análises de regressão linear para os dados agrupados mensalmente, anualmente e se considerando todos os dados do período, obtiveram-se os valores dos coeficientes "a" e "b" da equação de Angström-Prescott (Eq. 1):

$$
\mathrm{Rs}=\left(\mathrm{a}+\mathrm{b} \frac{\mathrm{n}}{\mathrm{N}}\right) \mathrm{Ra}
$$

em que:
Rs - radiação solar, $\mathrm{MJ} \mathrm{m}^{-2} \mathrm{~d}^{-1}$
$\mathrm{Ra}$ - radiação extraterrestre, $\mathrm{MJ} \mathrm{m}^{-2} \mathrm{~d}^{-1}$
$\mathrm{n}$ - número de horas de brilho solar, $\mathrm{h} \mathrm{d}^{-1}$
$\mathrm{N}$ - número máximo de horas de brilho solar, $\mathrm{h} \mathrm{d}^{-1}$ 
Para os coeficientes obtidos a partir dos dados mensais e anual, bem como para os valores estimados de Rs a partir de outros coeficientes, foram calculados os intervalos de confiança, a nível de $1 \%$ de probabilidade. Segundo Ferreira (2009), o intervalo de confiança é utilizado para indicar a confiabilidade de uma estimativa.

A partir dos dados de radiação (Rs) estimados pelas metodologias: a) coeficientes "a" e "b" mensais; b) coeficientes "a" e "b" anual; c) coeficientes "a" e "b" geral; d) coeficientes "a" e "b" propostos por Glover \& McCulloch (1958) $(a=0,29$ cos(latitude) e b $=0,52)$; e e) coeficientes " $a$ " e "b" propostos pela FAO ( $\mathrm{a}=0,25$ e $\mathrm{b}=0,50)$ (Allen et al., 1998), a evapotranspiração de referência foi estimada diariamente utilizando-se o método de Penman-Monteith FAO-56 (Allen et al., 1998) e, posteriormente, correlacionada com a ETo obtida a partir dos valores medidos de Rs, considerada referência. Ressalta-se que valores de Rs são utilizados nos cálculos das radiações de onda curta e onda longa, os quais permitem estimar o saldo de radiação, importante variável utilizada na estimativa da ETo.

Conforme metodologia apresentada por Barros et al. (2009) e Carvalho et al. (2006), foram estimados o erro-padrão da estimativa (SEE), o coeficiente de correlação (r) e o índice de concordância (d), proposto por Willmott (1981). Avaliou-se o desempenho das metodologias de estimativa dos coeficientes da equação Angströn-Prescott utilizando-se o índice de desempenho (c), correspondente à multiplicação do índice de "r" por "d", cuja descrição é apresentada por Camargo \& Sentelhas (1997).

\section{RESULTADOS E DISCUSSÃO}

A Figura 1 apresenta a distribuição dos valores médios de Rs e de ETo ao longo do ano, para o período de 2000 a 2007. Percebe-se a semelhança no comportamento da Rs em relação à ETo, sendo sua estimativa pelo método de Penmam-Monteith muito influenciada pelo radiação solar incidente (Carvalho et al., 2007). Os valores médios de Rs variaram de 7,35 a 26,20 $M J ~ m^{-2} \mathrm{~d}^{-1}$, sendo esses observados, respectivamente, nos dias

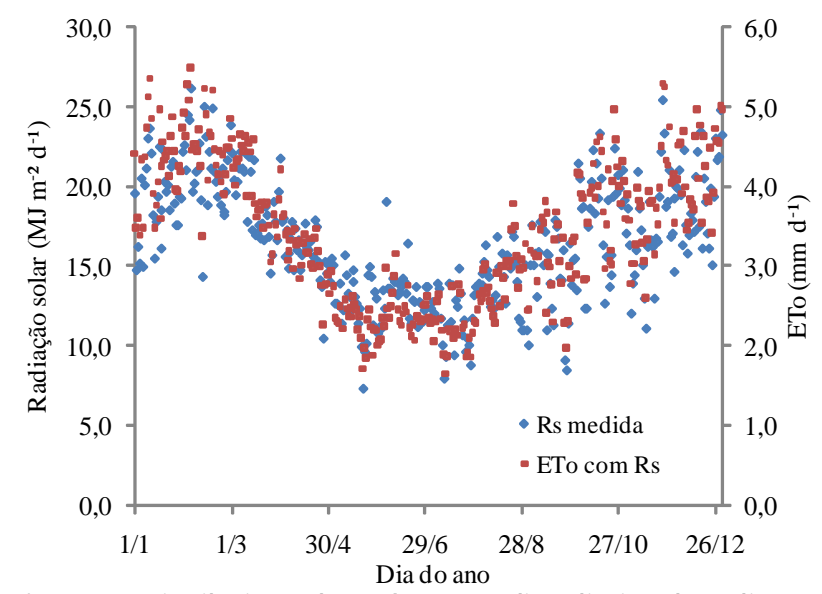

Figura 1. D istribuição dos valoresmédios diários de radiação solar (Rs) e evapotranspiração de referência (ETo), no período de 2000 a 2007, na estação Ecologia Agrícola
23/05 e 05/02; para a ETo, os valores médios variaram de 1,66 a $5,48 \mathrm{~mm} \mathrm{~d}^{-1}$, respectivamente, para os dias 13/07 e 05/02; os valores médios anuais observados para Rs e ETo, foram de $16,37 \mathrm{MJ} \mathrm{m}^{-2} \mathrm{~d}^{-1}$ e $3,38 \mathrm{~mm} \mathrm{~d}^{-1}$.

A Figura 2 apresenta a distribuição anual dos valores de $\mathrm{Rs} / \mathrm{Ra}$ em relação à $\mathrm{n} / \mathrm{N}$, considerando-se todos os dados disponíveis (Geral).

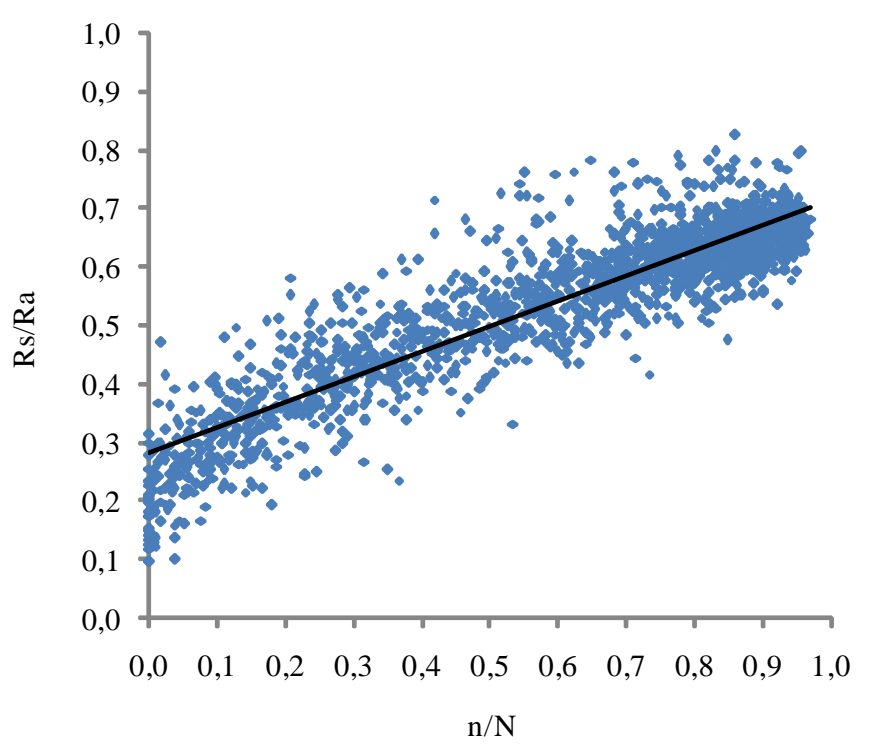

Figura 2. Distribuição diária da razão entre a radiação solar e a extraterrestre (Rs/Ra) e a razão insolação (n/N), no período de 2000 a 2007, na estação Ecologia Agrícola

Apesar da variabilidade, observa-se tendência de ajuste linear com relação à dependência entre essas grandezas, sendo obtido um coeficiente de determinação $\left(\mathrm{R}^{2}\right)$ de 0,82 . Dornelas et al. (2006) e Dallacort et al. (2004) encontraram, analisando a distribuição das mesmas variáveis, $\mathrm{R}^{2}$ de 0,81 e de 0,839 , respectivamente, para Brasília e para a região de Palotina, $\mathrm{PR}$.

A Tabela 1 apresenta o resultado das estimativas mensais, anual e geral dos coeficientes " $a$ " e "b" de Angströn-Prescott, tendo-se encontrado, para todas as análises, coeficientes de determinação acima de 0,81 . Os valores médios mensais de "a" variaram de 0,232 (agosto) a 0,299 (janeiro) e os valores médios mensais de "b" variaram de 0,397 (abril) a 0,504 (janeiro); os maiores valores de "a" compreendem a época do período chuvoso, momento em que a transmissividade da atmosfera é maior em virtude da redução da concentração de partículas suspensas (Blanco \& Sentelhas, 2002). Analisando os intervalos de confiança desses parâmetros e se considerando o nível de significância utilizado, é possível constatar que a variação observada na estimativa dos parâmetros foi relativamente pequena. De maneira semelhante aos resultados observados por Dornelas et al. (2006), nos meses que compreendem a estação seca foram calculados os maiores intervalos de confiança para os coeficientes "a" e "b"; nesses meses é comum o aumento de partículas sólidas na atmosfera em razão da menor umidade e, consequentemente, menor ocorrência de precipitações.

Quanto aos valores de radiação, é possível observar, na Tabela 1, que os valores medidos e calculados apresentaram o 
Tabela 1. Valores médios mensais, anual e de todo o período (geral) dos parâmetros da equação de Angstrom-Prescott, e seus respectivos coeficientes de determionação e val ores da radiação calculada e medida ( $M \mathrm{~J} \mathrm{~m}^{2} \mathrm{~d}^{-1}$ ), na estação Ecologia Agrícola

\begin{tabular}{cccccc}
\hline Mês & $\mathbf{a}$ & $\mathbf{b}$ & $\mathbf{R}^{2}$ & Rs calculada & Rs medida \\
Janeiro & $0,299 \pm 0,031$ & $0,430 \pm 0,043$ & 0,865 & $22,44 \pm 1,09$ & $22,09 \pm 1,28$ \\
Fevereiro & $0,266 \pm 0,029$ & $0,480 \pm 0,048$ & 0,834 & $22,51 \pm 1,08$ & $22,39 \pm 1,08$ \\
Março & $0,289 \pm 0,036$ & $0,427 \pm 0,036$ & 0,879 & $19,11 \pm 0,91$ & $19,17 \pm 1,05$ \\
Abril & $0,279 \pm 0,027$ & $0,397 \pm 0,057$ & 0,879 & $16,14 \pm 0,66$ & $16,04 \pm 0,74$ \\
Maio & $0,264 \pm 0,043$ & $0,441 \pm 0,061$ & 0,885 & $14,01 \pm 0,66$ & $13,75 \pm 0,82$ \\
J unho & $0,281 \pm 0,038$ & $0,428 \pm 0,072$ & 0,886 & $13,26 \pm 0,48$ & $13,71 \pm 0,71$ \\
Julho & $0,246 \pm 0,070$ & $0,455 \pm 0,084$ & 0,922 & $13,58 \pm 0,61$ & $13,48 \pm 0,73$ \\
Agosto & $0,232 \pm 0,070$ & $0,470 \pm 0,085$ & 0,888 & $15,02 \pm 0,80$ & $15,20 \pm 0,77$ \\
Setembro & $0,277 \pm 0,054$ & $0,468 \pm 0,057$ & 0,868 & $17,61 \pm 1,04$ & $16,83 \pm 1,31$ \\
Outubro & $0,277 \pm 0,044$ & $0,504 \pm 0,045$ & 0,881 & $20,94 \pm 1,09$ & $20,38 \pm 1,13$ \\
Novembro & $0,269 \pm 0,035$ & $0,489 \pm 0,048$ & 0,875 & $20,37 \pm 1,07$ & $19,54 \pm 1,29$ \\
Dezembro & $0,294 \pm 0,047$ & $0,495 \pm 0,050$ & 0,861 & $21,46 \pm 1,15$ & $21,67 \pm 1,37$ \\
\hline Anual & $0,295 \pm 0,038$ & $0,417 \pm 0,043$ & 0,812 & $18,12 \pm 0,33$ & $18,19 \pm 0,79$ \\
Geral & 0,282 & 0,433 & 0,820 & $17,99 \pm 0,29$ & $18,05 \pm 0,35$ \\
\hline
\end{tabular}

mesmo comportamento de variação; no período de abril a agosto foram calculados e medidos os menores valores de Rs e obtidos os menores valores de intervalo de confiança.

A Figura 3 apresenta os resultados das análises de correlação e o desempenho entre os valores de radiação medidos e os valores calculados, utilizando-se as diferentes metodologias propostas.

Observa-se um ajuste adequado para todas as metodologias, sendo o melhor desempenho (ótimo) obtido para a estimativa da radiação solar quando se utilizaram os coeficientes mensais da equação de Angström-Prescott (Figura 3A). Em complemento à Figura 3, os valores de erro padrão de estimativa variaram de 5,92 a $7,66 \mathrm{MJ} \mathrm{m}^{-2} \mathrm{~d}^{-1}$, sendo o maior valor correspondente à metodologia proposta por Glover \& McCulloch (1958), na qual o coeficiente "a" é obtido em função da latitude do local.

A Tabela 2 apresenta o resultado da análise mensal da estimativa da Rs através das diferentes metodologias. Observase que os índices de desempenho (c) variaram de 0,65 a 0,85. Com exceção da metodologia proposta pela FAO (mês de maio), os maiores valores deste índice foram encontrados no período de março a junho; para o mês de janeiro se encontraram os menores valores de "c", quando se utilizaram os coeficientes obtidos a partir da Latitude, FAO e dados gerais e anuais. Por outro lado, utilizando-se os coeficientes obtidos com os dados mensais, o menor índice de desempenho foi obtido para o mês de dezembro. Conforme observado na Tabela 1, os maiores índices de desempenho foram obtidos para o período seco.

O método de estimativa de Rs através da qual se utilizaram os coeficientes ajustados mensalmente, proporcionou os melhores desempenhos ao longo do ano, sendo este recomendado para o cálculo de Rs; este resultado também foi observado na análise anual, apresentada na Figura 3A.

Com exceção do método denominado Latitude, os menores valores de erro padrão de estimativa (SEE) foram observados para os meses secos; para este método, notou-se o maior SEE $\left(3,64 \mathrm{MJ} \mathrm{m}^{-2} \mathrm{~d}^{-1}\right)$ correspondente ao mês de janeiro enquanto o menor valor $\left(0,70 \mathrm{MJ} \mathrm{m}^{-2} \mathrm{~d}^{-1}\right)$ foi obtido para o mês de junho, utilizando-se coeficientes mensais.

A Figura 4 apresenta os resultados das análises de correlação entre os valores de ETo calculados a partir dos valores de Rs medidos e calculados utilizando-se as diferentes metodologias propostas. Observa-se que todas as estimativas de ETo

Tabela 2. Valores mensais de erro padrão de estimativa (SEE - $\mathrm{mm} \mathrm{d}^{-1}$ ) e índice de desempenho (c) entre valores diários de radiação solar ( $\left(\mathrm{M} J \mathrm{~m}^{2} \mathrm{~d}^{-1}\right)$ obtidos por diferentes metodologias para determinação dos coeficientes da equação de AngströmPrescott

\begin{tabular}{|c|c|c|c|c|c|c|c|c|c|c|c|c|c|c|c|}
\hline \multirow{3}{*}{ Mês } & \multicolumn{15}{|c|}{ Métodos de estimativa de Rs } \\
\hline & \multicolumn{3}{|c|}{ Mensais } & \multicolumn{3}{|c|}{ Geral } & \multicolumn{3}{|c|}{ Anual } & \multicolumn{3}{|c|}{ Latitude* } & \multicolumn{3}{|c|}{ FAO } \\
\hline & SEE & c & D & SEE & c & D & SEE & c & D & SEE & c & D & SEE & c & D \\
\hline J an & 2,04 & 0,72 & $B$ & 3,24 & 0,65 & $M$ & 3,36 & 0,65 & M & 3,64 & 0,65 & M & 3,14 & 0,66 & B \\
\hline $\mathrm{Fev}$ & 1,49 & 0,76 & MB & 1,58 & 0,76 & MB & 1,53 & 0,76 & MB & 1,64 & 0,75 & B & 1,54 & 0,75 & B \\
\hline Mar & 1,13 & 0,83 & MB & 1,17 & 0,82 & MB & 1,12 & 0,83 & MB & 1,47 & 0,80 & $M B$ & 1,18 & 0,82 & MB \\
\hline$A b r$ & 0,82 & 0,85 & MB & 1,18 & 0,83 & MB & 1,23 & 0,84 & MB & 2,28 & 0,76 & MB & 1,47 & 0,80 & MB \\
\hline Mai & 0,88 & 0,83 & MB & 1,02 & 0,82 & MB & 1,07 & 0,82 & MB & 1,73 & 0,78 & MB & 1,76 & 0,73 & B \\
\hline J un & 0,70 & 0,84 & MB & 0,77 & 0,84 & MB & 0,79 & 0,84 & MB & 1,66 & 0,80 & MB & 1,05 & 0,83 & MB \\
\hline Jul & 1,02 & 0,82 & MB & 1,35 & 0,79 & MB & 1,41 & 0,78 & MB & 2,07 & 0,74 & B & 1,48 & 0,79 & MB \\
\hline Ago & 1,02 & 0,78 & MB & 1,38 & 0,75 & $B$ & $\begin{array}{l}1,1 \\
1,44\end{array}$ & 0,74 & B & 2,26 & 0,70 & B & 1,57 & 0,75 & B \\
\hline Set & 1,91 & 0,76 & MB & 1,72 & 0,77 & MB & $\begin{array}{l}1,85 \\
1,85\end{array}$ & 0,76 & MB & 2,21 & 0,75 & B & 1,64 & 0,77 & MB \\
\hline Out & 2,10 & 0,72 & $B$ & 1,78 & 0,75 & B & 1,85 & 0,73 & B & 2,04 & 0,73 & B & 1,70 & 0,77 & MB \\
\hline Nov & 2,25 & 0,77 & $M B$ & 2,71 & 0,76 & $M B$ & 2,85 & 0,75 & B & 3,12 & 0,75 & B & 2,61 & 0,78 & MB \\
\hline Dez & 1,88 & 0,68 & B & 2,05 & 0,68 & B & 1,94 & 0,68 & B & 1,83 & 0,68 & B & 2,22 & 0,67 & B \\
\hline Média & 1,44 & & & 1,66 & & & 1,70 & & & 2,16 & & & 1,78 & & \\
\hline
\end{tabular}

* proposto por Glover \& McCulloch (1958)

$\mathrm{D}=$ desempenho: $\mathrm{M}$ - mediano; $\mathrm{B}$ - bom; $\mathrm{MB}$ - muito bom 
A.

B.
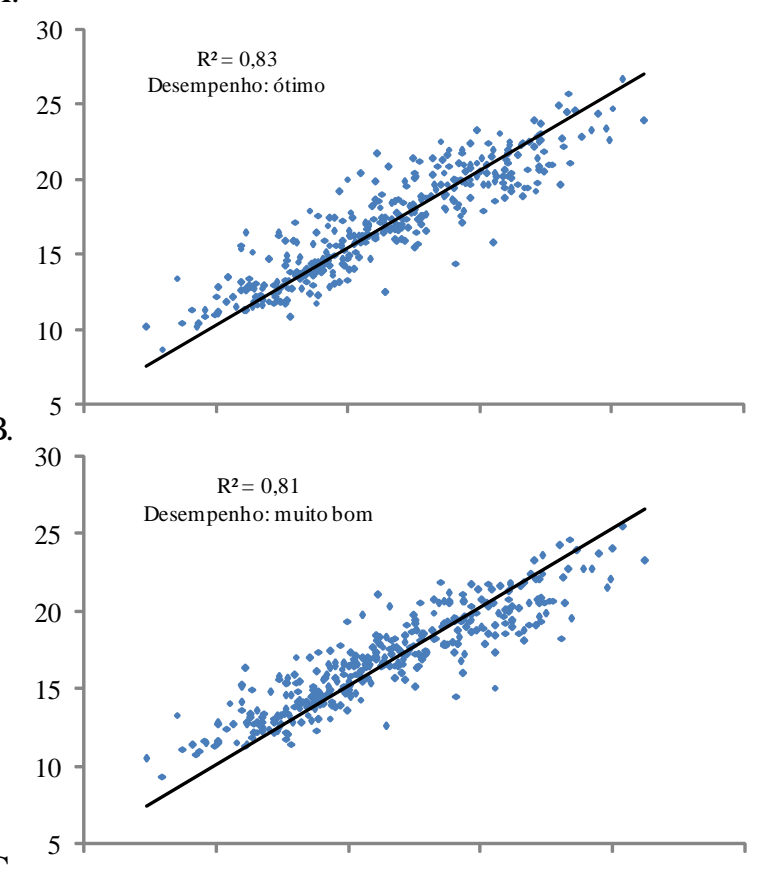

C.

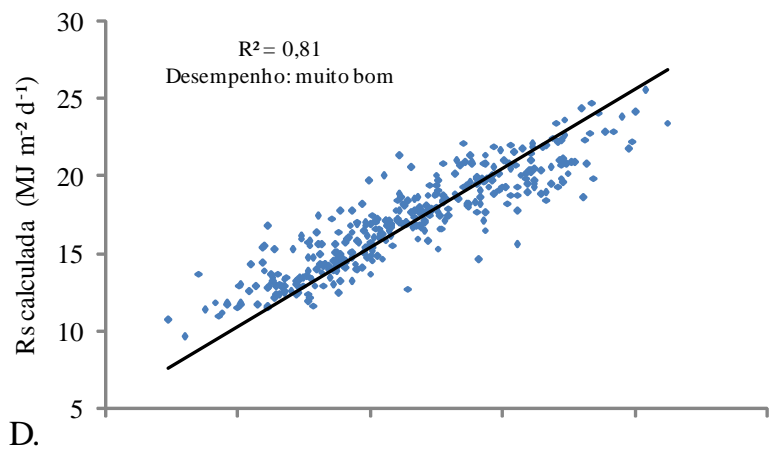

D.

E.
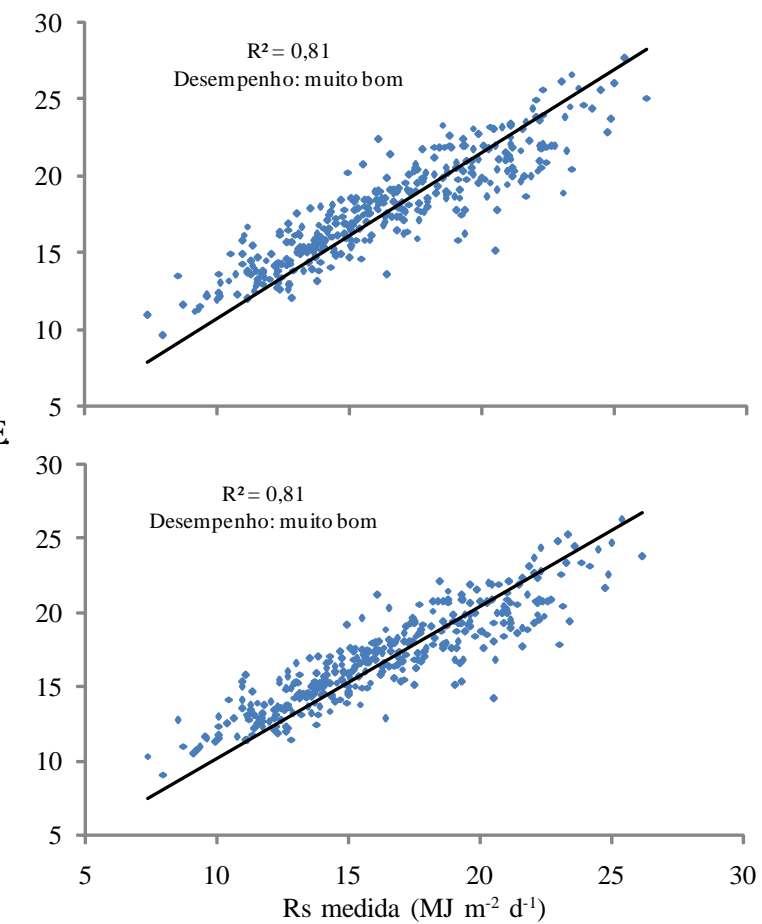

Figura 3. Comparação dos valores diários de radiação solar (Rs) medidos e calculados pelas metodologias: A. mensal; B. geral; C. anual; D. latitude; E. FAO
A.

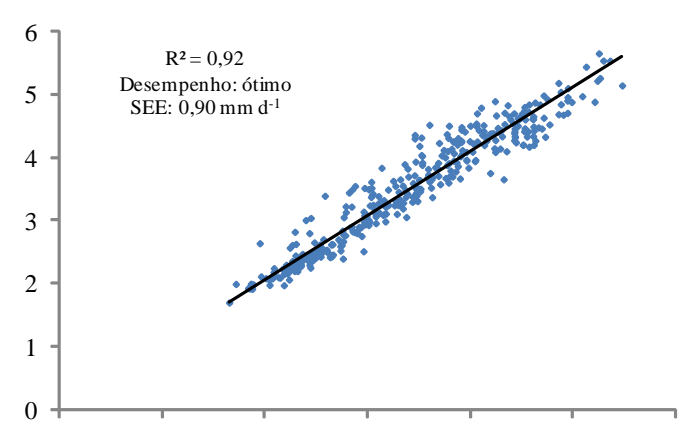

B.

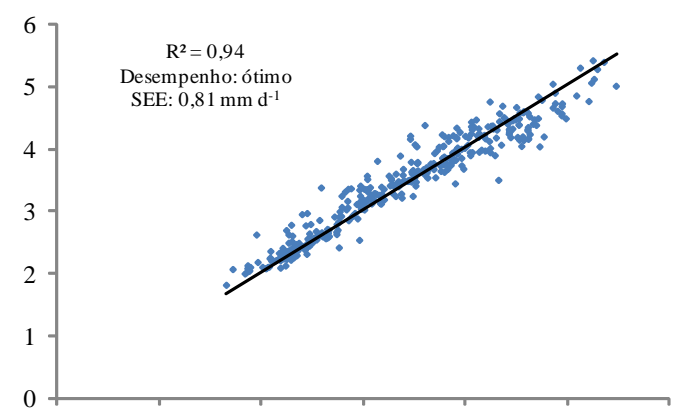

C.

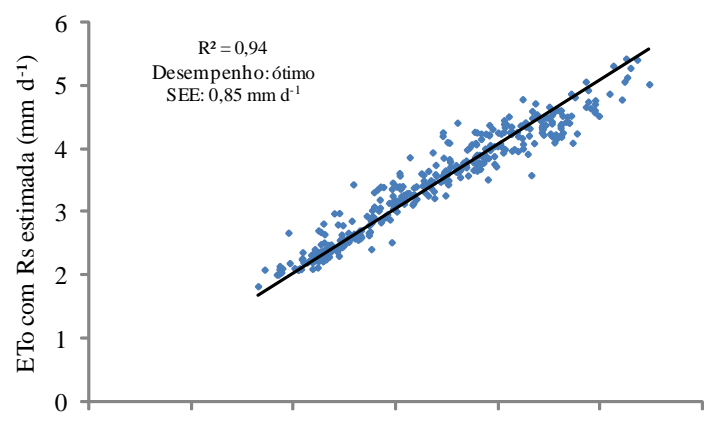

D.

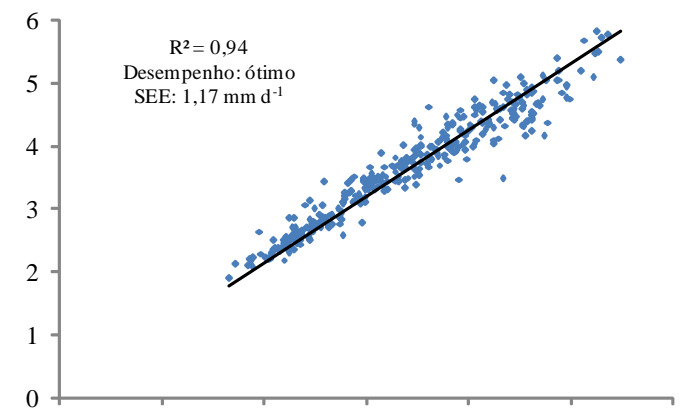

E

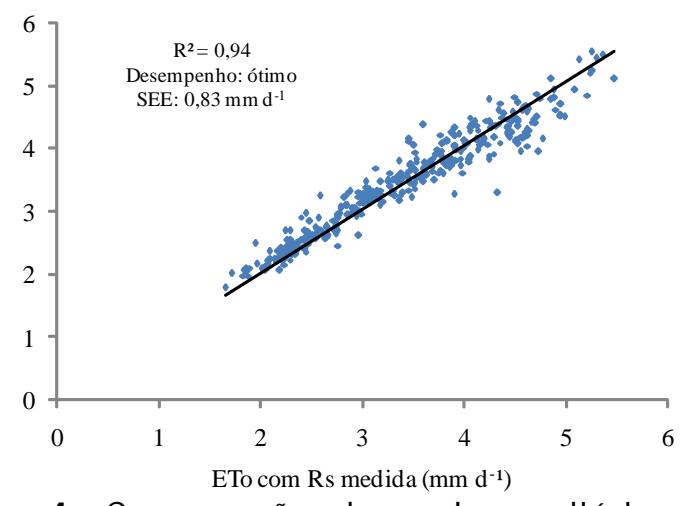

Figura 4. Comparação dos valores diários de evapotranspiração de referência (ETo) obtidoscom valores de Rs medidos e cal cul ados pel as metodologias: A. mensal; B. geral; C. anual; D. latitude; E. FAO 
Tabela 3. Valores mensais de erro padrão de estimativa (SEE - $\mathrm{mm} \mathrm{d}^{-1}$ ) e índice de desempenho (c) entre valores diários de ETo obtidos por P-M utilizando-se dados medidos e diferentes meto dologias de estimativa de Rs

\begin{tabular}{|c|c|c|c|c|c|c|c|c|c|c|c|c|c|c|c|}
\hline \multirow{3}{*}{ Mês } & \multicolumn{15}{|c|}{ Métodos de estimativa de Rs } \\
\hline & \multicolumn{3}{|c|}{ Mensais } & \multicolumn{3}{|c|}{ Geral } & \multicolumn{3}{|c|}{ Anual } & \multicolumn{3}{|c|}{ Latitude* } & \multicolumn{3}{|c|}{ FAO } \\
\hline & SEE & c & D & SEE & c & D & SEE & c & D & SEE & c & D & SEE & C & D \\
\hline Jan & 0,43 & 0,60 & $\mathrm{~S}$ & 0,36 & 0,64 & M & 0,38 & 0,63 & M & 0,51 & 0,56 & $S$ & 0,39 & 0,62 & $M$ \\
\hline Fev & 0,25 & 0,76 & MB & 0,26 & 0,76 & MB & 0,25 & 0,77 & MB & 0,31 & 0,72 & $B$ & 0,25 & 0,75 & B \\
\hline Mar & 0,17 & 0,91 & 0 & 0,18 & 0,91 & 0 & 0,17 & 0,91 & 0 & 0,27 & 0,87 & $M B$ & 0,18 & 0,90 & 0 \\
\hline $\mathrm{Abr}$ & 0,15 & 0,88 & 0 & 0,16 & 0,88 & 0 & 0,17 & 0,87 & 0 & 0,39 & 0,69 & $B$ & 0,21 & 0,83 & MB \\
\hline Mai & 0,10 & 0,90 & 0 & 0,11 & 0,89 & 0 & 0,13 & 0,87 & 0 & 0,25 & 0,77 & $M B$ & 0,13 & 0,89 & 0 \\
\hline J un & 0,12 & 0,85 & MB & 0,11 & 0,85 & MB & 0,12 & 0,85 & MB & 0,22 & 0,77 & $M B$ & 0,12 & 0,85 & MB \\
\hline Jul & 0,10 & 0,92 & 0 & 0,12 & 0,90 & 0 & 0,13 & 0,88 & MB & 0,26 & 0,77 & MB & 0,14 & 0,89 & 0 \\
\hline Ago & 0,13 & 0,92 & 0 & 0,15 & 0,90 & 0 & 0,16 & 0,89 & 0 & 0,31 & 0,78 & $M B$ & 0,18 & 0,89 & 0 \\
\hline Set & 0,30 & 0,81 & $M B$ & 0,26 & 0,84 & MB & 0,10 & 0,81 & MB & 0,36 & 0,77 & $M B$ & 0,09 & 0,85 & MB \\
\hline Out & 0,33 & 0,89 & 0 & 0,25 & 0,90 & 0 & 0,27 & 0,89 & 0 & 0,32 & 0,88 & 0 & 0,23 & 0,92 & 0 \\
\hline Nov & 0,35 & 0,86 & 0 & 0,31 & 0,87 & 0 & 0,35 & 0,85 & MB & 0,42 & 0,83 & MB & 0,27 & 0,89 & 0 \\
\hline Dez & 0,38 & 0,64 & $M$ & 0,33 & 0,63 & $M$ & 0,32 & 0,64 & $M$ & 0,32 & 0,66 & B & 0,36 & 0,62 & $M$ \\
\hline Média & 0,23 & & & 0,22 & & & 0,21 & & & 0,33 & & & 0,21 & & \\
\hline
\end{tabular}

* proposto por Glover \& McCulloch (1958)

$\mathrm{D}=$ Desempenho: $\mathrm{S}$ - sofrível; $\mathrm{M}$ - mediano; $\mathrm{B}$ - bom; $\mathrm{MB}$ - muito bom; 0 - ótimo

obtiveram índice de desempenho ótimo, independente da metodologia utilizada na obtenção dos coeficientes da equação de Angström-Prescott.

Com relação às análises apresentadas na Figura 4 , os erros padrões de estimativa encontrados foram de 0,$90 ; 0,81 ; 0,85$; 1,17 e $0,83 \mathrm{~mm} \mathrm{~d}^{-1}$, respectivamente, para as metodologias denominadas mensal, geral, anual, latitude e FAO.

A Tabela 3 apresenta o resultado da análise mensal da estimativa da ETo, através das diferentes metodologias de cálculo da Rs. Apesar da análise apresentada na Figura 4 indicar ótimo desempenho para os diferentes métodos de cálculo da Rs, a análise mensal indica variações expressivas entre os métodos. Os índices de desempenho (c) variaram de 0,56 a 0,92 , indicando comportamento oscilando de sofrível a ótimo (Camargo \& Sentelhas, 1997).

Da mesma forma como observado na Tabela 2, os menores índices de desempenho foram obtidos para os meses de dezembro e janeiro, sendo para este último obtidos valores de "c" considerados sofríveis para as metodologias nas quais foram utilizados dados de Latitude e Mensais. Por outro lado e seguindo o comportamento observado para a radiação solar, os meses mais secos do ano foram aqueles em que se obtiveram os maiores valores de índice de desempenho; este resultado se torna relevante visto que, como a região é caracterizada por um inverno seco, o uso dessas metodologias para estimativa da lâmina de irrigação a partir de estimativas de Rs e ETo, não se torna inviável.

Na Tabela 3 se observa que o SEE apresentou valores que variaram de $0,09 \mathrm{~mm} \mathrm{~d}^{-1}$ (FAO - setembro) a 0,51 $\mathrm{mm} \mathrm{d}^{-1}$ (Latitude - janeiro). Analisando a média, tem-se que os SEE para ETo estimada por diferentes metodologias de cálculo da Rs, não apresentaram variação expressiva, com exceção do método denominado Latitude; entretanto, analisando o período seco (abril-agosto), constata-se que os coeficientes obtidos a partir da análise mensal dos dados de Rs foram aqueles que proporcionaram menor $\operatorname{SEE}\left(0,12 \mathrm{~mm} \mathrm{~d}^{-1}\right)$.
Apesar do desempenho aceitável da metodologia de estimativa de Rs proposta pela FAO (Allen et al., 1998), que é amplamente difundida, pode-se constatar que a utilização de metodologias a partir de estimativas locais de "a" e "b" apresentou resultados mais satisfatórios para o cálculo da evapotranspiração em Seropédica,RJ. Dornellas et al. (2006), analisando os coeficientes da equação de AngströmPrescott para Brasília, concluíram que as estimativas de ETo não diferiram dos valores observados tendo-se constatado ótimo desempenho, independentemente do coeficiente utilizado.

\section{CONCLusÕES}

1. O maior desempenho para a estimativa da radiação solar global pela equação de Angström-Prescott, foi obtido com a utilização dos coeficientes médios mensais de a e b; contudo, os índices de desempenho foram semelhantes quando se adotaram os coeficientes médios nos períodos anuais, gerais e aqueles utilizando a latitude e os propostos pela FAO.

2. As estimativas de ETo apresentaram índice de desempenho ótimo, independente da metodologia utilizada na obtenção dos coeficientes da equação de Angström-Prescott para o cálculo de Rs; no entanto, a partir da análise de estimativas locais para o cálculo de Rs utilizando-se coeficientes médios mensais, observa-se que os resultados são mais satisfatórios em relação aos coeficientes propostos pela FAO.

\section{Agradecimento}

A Coordenação Geral de Agrometeorologia do Instituto Nacional de Meteorologia (INMET), na pessoa do Dr. Alaor Moacyr Dall’Antonia Jr., pela disponibilização dos dados meteorológicos da estação automática. 


\section{LITERATURA CITADA}

Allen, R. G.; Pereira, L. S.; Raes, D.; Smith, M. Crop evapotranspiration-guidelines for computing crop water requirements. Rome: FAO, 1998. 297p. Irrigation and drainage paper, 56.

Almorox, J.; Benito, M.; Hontoria, C. Estimation of global solar radiation in Venezuela. INCI, v.33, p.280-283, 2008.

Azevedo, P. V. de; Leitão, M. de M. V. B. R.; Souza, I. F. de; Maciel, G. F. Balanço de radiação sobre culturas irrigadas no semi-árido do Nordeste do Brasil. Revista Brasileira de Meteorologia, v.5, p.403-410, 1990.

Barros, V. R.; Souza, A. P.; Carvalho, D. F.; Silva, L. D. B. Evapotranspiração de referência na região de SeropédicaRJ, utilizando lisímetro de pesagem e modelos matemáticos. Revista Brasileira de Ciências Agrárias, v.4, p.198-203, 2009.

Blanco, F. F.; Sentelhas, P. C. Coeficientes da equação de Angströn-Prescott para estimativa da insolação para Piracicaba, SP. Revista Brasileira de Agrometeorologia, v.10, p.295-300, 2002.

Camargo, A. P.; Sentelhas, P. C. Avaliação do desempenho de diferentes métodos de estimativa da evapotranspiração potencial no Estado de São Paulo, Brasil. Revista Brasileira de Agrometeorologia, v.5, p.89-97, 1997.

Campello Júnior, J. H. Relação sazonal entre radiação solar global e insolação no sudoeste da Amazônia. Revista Brasileira de Agrometeorologia, v.6, p.193-199, 1998.

Carvalho, D. F.; Silva, L. D. B.; Folegatti, M. V.; Costa, J. R.; Cruz, F. A. Avaliação da evapotranspiração de referência na região de Seropédica, RJ, utilizando lisímetro de pesagem. Revista Brasileira de Agrometeorologia, v.14, p.108-116, 2006.

Carvalho, D. F.; Silva, L. D. B.; Guerra, J. G. M.; Cruz, F. A.; Souza, A. P. Instalação, calibração e funcionamento de um lisímetro de pesagem. Engenharia Agrícola, v.27, p.363-372, 2007.
Cervellini, A.; Salati, E.; Godoy, H. Estimativa da distribuição da energia solar no Estado de São Paulo. Bragantia, v.23, p.31-39, 1966.

Dallacort, R.; Freitas, P. S. L. de; Gonçalves, A. C. A.; Rezende, R.; Bertonha, A.; Silva, F. F. da; Trintinalha, M. A. Determinação dos coeficientes da equação de Angströn para a região de Palotina, estado do Paraná. Acta Scientiarum. Agronomy, v.26, p.329-336, 2004.

Dantas, A. A.; Carvalho, L. G. de; Ferreira, E. Estimativa da radiação solar global para a região de Lavras, MG. Ciência e Agrotecnologia, v.27, p.1260-1263, 2003.

Dornelas, K. D. e S.; Silva, C. L. da; Oliveira, C. A. da S. Coeficientes médios da equação de Angström-Prescott, radiação solar e evapotranspiração de referência em Brasília. Pesquisa Agropecuária Brasileira, v.41, p.12131219, 2006.

Ferreira, D. F. Estatística básica. 2a. ed. Lavras: UFLA, 2009. $664 p$.

Glover, J.; McCulloch, J. S. G. The empirical relation between solar radiation and hours of sunshine. The Quarterly Journal of the Royal Meteorological Society, v.84, p.172$175,1958$.

Liu, X.; Mei, X.; Li, Y.; Wang, Q.; Zhang, Y.; Porter, J. R. Variation in reference crop evapotranspiration caused by the Angström-Prescott coefficient: Locally calibrated versus the FAO recommended. Agricultural Water Management, v.96, p.1137-1145, 2009a.

Liu, X.; Mei, X.; Li, Y.; Zhang, Y.; Wang, Q.; Jensen, J. R.; Porter, J. R. Calibration of the Angström-Prescott coefficients ( $\mathrm{a}, \mathrm{b})$ under different time scales and their impacts in estimating global solar radiation in the Yellow River basin. Agricultural and Forest Meteorology, v.149, p.696-710, 2009b.

Valiati, M. I.; Ricieri, R. P. Estimativa da irradiação solar global com partição mensal e sazonal para região de Cascavel PR. Engenharia Agrícola, v.25, p.76-85, 2005.

Willmott, C. J. On the validation of model. Physical Geography, v.2,p.184-194, 1981. 